\title{
Validation of a recording system for computer pointing device activity
}

\author{
Yaw-Huei Hwang ${ }^{\mathrm{a}, \mathrm{b}, *}$, Ching-Hsyang Wang ${ }^{\mathrm{a}}$, Huey-Wen Liang ${ }^{\mathrm{a}, \mathrm{c}}$, Jer-Junn Luh $^{\mathrm{d}}$ \\ a Institute of Occupational Medicine and Industrial Hygiene, College of Public Health, National Taiwan University, Rm. 735, \#17, Xu-Zhou Rd., Taipei, Taiwan, ROC \\ ${ }^{\mathrm{b}}$ Department of Public Health, College of Public Health, National Taiwan University, Rm. 735, \#17, Xu-Zhou Rd., Taipei, Taiwan, ROC \\ ${ }^{\mathrm{c}}$ Department of Physical Medicine and Rehabilitation, National Taiwan University Hospital, National Taiwan University College of Medicine, \\ No. 1, Chang-Teh St., Taipei, Taiwan, ROC \\ ${ }^{\mathrm{d}}$ School and Graduate Institute of Physical Therapy, College of Medicine, National Taiwan University, Rm. 320, \#17, Xu-Zhou Rd., Taipei, Taiwan, ROC
}

\section{A R T I C L E I N F O}

\section{Article history:}

Received 16 October 2007

Received in revised form

2 December 2008

Accepted 11 January 2009

Available online $\mathrm{xxx}$

\section{Keywords:}

Mlog

Computer work

Pointing device

Validation

\begin{abstract}
A B S T R A C T
This study reports on the development of the pointing device activity monitoring software, Mlog, which is used to assess elements of computer use such as pointing device use time. The Mlog monitoring program was written with Virtual $\mathrm{C}_{+}+$and is $1.59 \mathrm{MB}$ in size. It is applicable for computers equipped with computer processing units (CPUs) of $1 \mathrm{GHz}$ or faster, and records pointing device activity at the millisecond level. The accuracy, precision, and reliability of the Mlog monitoring program were tested by comparing Mlog-recorded activity time intervals, for example, the time interval between pressing and releasing the pointing device key, against oscilloscope-recorded pointing device electric signals. For most tested time intervals, the Mlog monitoring program's margin of error for accuracy was within $\pm 0.50 \%$, and it boasted precision error rate of less than $1.00 \%$. Correlation coefficients between the Mlog recordings and the references were all greater than 0.99905 , with the highest values observed for dragging actions. For most tested time intervals, the results of the reliability test showed that their intraclass correlation coefficients were higher than 0.95 , with few less than 0.90 for those with relatively longer tested time intervals. Linear regression analyses indicated that the pointing device clicking action time intervals recorded by the Mlog monitoring program were very close to the reference values and not affected by CPU speed and memory capacity, computer system loadings of software programs in operation, or types of pointing device key action. In conclusion, the Mlog monitoring program was validated as an accurate tool for monitoring pointing device activity during computer work. Future study is warranted to examine the influences of idle time threshold for electronic activity monitoring, passive computer tasks, and job type on the performance of the program in field studies.

Relevance to industry: The Mlog monitoring program was validated as accurate in measuring pointing device activity during computer work, providing an indirect and supplemental exposure indices for those traditional ergonomic measurements for physical loading assessment.
\end{abstract}

(c) 2009 Elsevier B.V. All rights reserved.

\section{Introduction}

It has been recognized that computer use is highly associated with occupational musculoskeletal symptoms and disorders (Bergqvist et al., 1995; Kimura et al., 2007; Nakazawa et al., 2002; Sauter et al., 1991; Sillanpaa et al., 2003; Wahlstrom, 2005). Other computerrelated adverse health outcomes include eye irritation (Blehm et al., 2005; Murata et al., 1991), reproduction hazard (Koren, 2003; Shaw, 2001), dermatitis (Bergdahl et al., 2004), anxiety (Smith, 1997) etc. Objective data on exposure to physical risk factors for musculoskeletal

\footnotetext{
* Corresponding author. Institute of Occupational Medicine and Industrial Hygiene, College of Public Health, National Taiwan University, Rm. 735, \#17, Xu-Zhou Rd., Taipei 10055, Taiwan, ROC. Tel./fax: +886 233668081.

E-mail address: yhhwang@ntu.edu.tw (Y.-H. Hwang).
}

disorders (MSDs) are commonly assessed using tools such as 3-D motion analysis systems, electromyography, electrogoniometers, and force sensors (Dennerlein and Johnson, 2006; Jensen et al., 1999; Johnson et al., 2000; Jonsson et al., 2007; Karlqvist et al., 1998; Keir et al., 1999; Kotani et al., 2007; Lee et al., in press; Rempel et al., 1997; Simoneau et al., 2003). Meanwhile, questionnaires are frequently used as a subjective measure to evaluate physical and psychological risk factors (Faucett and Rempel, 1994).

Most objective measurement tools need to attach markers or data loggers to subjects, potentially altering and restricting their movements. Therefore, the representativeness and accuracy of these measurements are often compromised (Allison, 2003). In addition, the relatively high cost of measuring equipment, as well as complicated set-up, calibration, and statistical analysis procedures, also require the expenditure of a great deal of resources and

0169-8141/\$ - see front matter (c) 2009 Elsevier B.V. All rights reserved. doi:10.1016/j.ergon.2009.01.007 
manpower. As a result, it is difficult to obtain longitudinal exposure data from large samples of video display terminal (VDT) users.

On the other hand, the interactions among individual, physical, psychological, environmental, and organizational factors closely influence the incidence of MSDs (Faucett and Rempel, 1994; Homan and Armstrong, 2003). However, it is complicated to measure these psychological or physiological risk factors for MSDs in the workplace, and, furthermore, it is difficult to evaluate them simultaneously. Therefore, an objective, comprehensive and scientifically sound indicator for computer work exposure - such as physical work loading measured in terms of use time and frequency, as well as by type of pointing device activity - is warranted in a large scale ergonomic population-based study.

Due to the prevalence of Internet use and graphical user interfaces for human-computer interaction, input devices are indispensable to computer work. To date, certain electronic activity monitoring systems have been developed for the estimation of total computer, keyboard, and mouse use time. Some of these physically pass electronic signals from keyboards and pointing devices to external data acquisition systems (Homan and Armstrong, 2003; Johnson et al., 2000). Some commercial programs can record and calculate real-time input device use duration and typing speed during computer use, where an example of such programs is WorkPace (Wellnomics Ltd., New Zealand, Christchurch). A previous study by the authors reported on the development of a compact and fully functional keyboard activity recording software program, KBlog (Chang et al., 2004), which records detailed temporal information about each signal keystroke. To promote our ability to further record detailed pointing device activity in accompaniment with the information that can be culled from KBlog monitoring program, the present study aims to validate the recording software program Mlog for pointing device activity monitoring during computer work. Mouse operation will be used as an example.

\section{Materials and methods}

\subsection{Technical characteristics of Mlog monitoring program}

A pointing device translates the mechanical movement of the device itself and its keys into digital signals communicated to the PC host. Every time a pointing device key is pressed, the mechanical switch underneath the key is activated to generate a voltage change detected by a controller chip inside the pointing device that codes the change into a digital signal. Subsequently, the pointing device controller chip transmits the digital message to the host. The operating system then receives, calculates and records the signal. Since the Windows operating system is not a real-time system, there is a potential time lag between the moment when a signal is sent by the pointing device and the time at which the operating system processes the signal.

There are four lines inside the wire connecting the pointing device to the computer; these function as the data line, clock line, ground line and $+5 \mathrm{~V}$ power supply. An oscilloscope was used in this study to monitor the voltage change for the data line connecting the pointing device and the host to indicate the electronic signal input generated by the pointing device. This information was then used as a reference for comparison with the Mlog monitoring data.

The software program used in the present study, Mlog, was designed to record pointing device activity during computer use. It was written with Virtual $\mathrm{C}++$, has a size of $1.59 \mathrm{MB}$, and is applicable for computers equipped with computer processing units (CPUs) of $1 \mathrm{GHz}$ or faster. After being installed into a computer system, the Mlog monitoring program operates based on an interface with icon selection to record pointing device activity. There is no need for additional hardware to be used for this program to function.
Since there are a variety of uses for pointing devices, the present study employed a mouse with two keys as an example in order to evaluate the performance of Mlog monitoring program. During the monitoring period, recorded data were written into a text file and named according to the date when the file was created, including 15 numbers indicating the date (year, month, date) and time (hour, minute, second) (Fig. 1). A logging data string contained 9 consecutive variables representing the data serial number $(\mathrm{SN})$, hour, minute, second and millisecond of logging time, $X-Y$ coordinates (with the left-bottom corner of the monitor screen as the origin), pointing device activity (defined in the following section), and time interval between pointing device key press and release (Fig. 1).

Pointing device activity type is recorded as moving action $<\mathrm{M}>$, press-down of the left key $<\mathrm{LD}>$, release of the left key $<\mathrm{LU}>$, pressdown of the right key $<\mathrm{RD}>$, release of the right key $<\mathrm{RU}>$, and the continuous action of holding a key $<\mathrm{H}>$. Calculations of the time intervals for pointing device action varied by the type of pointing device activity, as illustrated in Fig. 1. The clicking action time interval was defined as the difference between recorded pressdown and release times for a specific pointing device key. The dragging time was obtained in the same way, except that there were a few time records marked as action $<\mathrm{H}>$ were required between the recorded press-down and release times of a specific pointing device key; these indicated that it was a dragging action. Meanwhile, pointing device moving action was recorded once the cursor location on the computer screen was changed by an amount equal to or greater than one resolution on either the $X$ or $Y$ axis. The cursor locations were also scanned and registered based on the computer's electronic signal transmission rate - for example, $15.6 \mathrm{~ms}$ as the default value for the computer settings used in the present study. As for the time interval for moving action, it was calculated as the time difference between the first and the last data points of a series of consecutive recorded times marked as moving action $<\mathrm{M}>$.

\subsection{Accuracy and precision of the Mlog monitoring program}

An oscilloscope was used in the evaluation of the Mlog monitoring program. It was calibrated prior to the testing procedure to meet the requirements for accuracy: time error of less than $0.02 \%$. Since the testing procedure covers time periods ranging from 10 milliseconds ( $\mathrm{ms}$ ) to $10,000 \mathrm{~ms}$, a maximal oscilloscope time error ranging from $0.005 \mathrm{~ms}$ to $0.05 \mathrm{~ms}$ was considered acceptable. During the evaluation period, digital signals transmitted from the pointing device to the PC host were also collected by the oscilloscope via a cable connecting it to the pointing device transmission line. Every time the pointing device moved or a pointing device key was pressed and released, the oscilloscope detected the respective voltage change and displayed it on the screen of oscilloscope (Fig. 2). Manually pausing the oscilloscope freezes the changing waveform on the screen; thus, a specific time interval can be obtained by calculating the time period between the firing times of wave peaks on the screen generated by pressing and releasing the pointing device key. These measurements were used as references in the following evaluation of accuracy and precision for Mlog monitoring.

In the evaluation of the Mlog monitoring program performance, this monitoring software was first installed in the directory of C:|Program Files $\mid$ Mlog, and the pointing device was operated in Microsoft Word. The time intervals for pointing device actions were logged by the oscilloscope and the Mlog monitoring program, with both manually calculated and recorded. Eight designated time intervals of pointing device action were used for the evaluation, covering the time intervals from those for clicking actions to those for dragging and/or moving actions $(100 \mathrm{~ms}, 200 \mathrm{~ms}, 300 \mathrm{~ms}$, $450 \mathrm{~ms}, 1000 \mathrm{~ms}, 2000 \mathrm{~ms}, 3000 \mathrm{~ms}$, and 10,000 ms). For each of the specified time interval levels, one of the authors repeatedly 
a

\begin{tabular}{|c|c|c|c|c|c|c|c|c|c|}
\hline SN & hour & $\min$ & $\mathrm{sec}$ & $\mathrm{msec}$ & $X$ & Y & act & interval & \multirow{5}{*}{$\begin{array}{l}\text { Moving Time } \\
\text { Interval }\end{array}$} \\
\hline 0000001 & 00 & 00 & 00 & 159 & 1148 & 0796 & M & 00000000 & \\
\hline 0000002 & 00 & 00 & 00 & 175 & 1148 & 0797 & M & 00000016 & \\
\hline 0000003 & 00 & 00 & 00 & 191 & 1149 & 0799 & M & 00000032 & \\
\hline 0000004 & 00 & 00 & 00 & 207 & 1151 & 0801 & M & 00000048 & \\
\hline 0017183 & 00 & 06 & 47 & 208 & 0148 & 0320 & DR & 00000366 & \multirow{8}{*}{$\begin{array}{l}\text { Dragging Time } \\
\text { Interval }\end{array}$} \\
\hline 0017184 & 00 & 06 & 47 & 218 & 0147 & 0320 & $\mathrm{H}$ & 00000010 & \\
\hline 0017185 & 00 & 06 & 47 & 245 & 0147 & 0321 & $\mathrm{H}$ & 00000027 & \\
\hline 0017186 & 00 & 06 & 47 & 273 & 0146 & 0321 & $\mathrm{H}$ & 00000028 & \\
\hline 0017187 & 00 & 06 & 47 & 323 & 0146 & 0322 & $\mathrm{H}$ & 00000050 & \\
\hline 0017188 & 00 & 06 & 47 & 346 & 0145 & 0322 & $\mathrm{H}$ & 00000023 & \\
\hline 0017189 & 00 & 06 & 47 & 356 & 0145 & 0323 & $\mathrm{H}$ & 00000010 & \\
\hline 0017190 & 00 & 06 & 47 & 367 & 0145 & 0323 & UR & 00000159 & \\
\hline 0152323 & 01 & 01 & 09 & 064 & 1148 & 0837 & DL & 00001179 & Left Key Clicking \\
\hline 0152324 & 01 & 01 & 09 & 199 & 1148 & 0837 & UL & 00000135 & Time Interval \\
\hline 0152262 & 01 & 01 & 07 & 885 & 1111 & 0941 & DR & 00011573 & Right Key Clicking \\
\hline 0152263 & 01 & 01 & 08 & 012 & 1111 & 0941 & UR & 00000127 & Time Interval \\
\hline
\end{tabular}

Example of data logging system and time interval calculations for clicking, dragging, and moving actions.

b

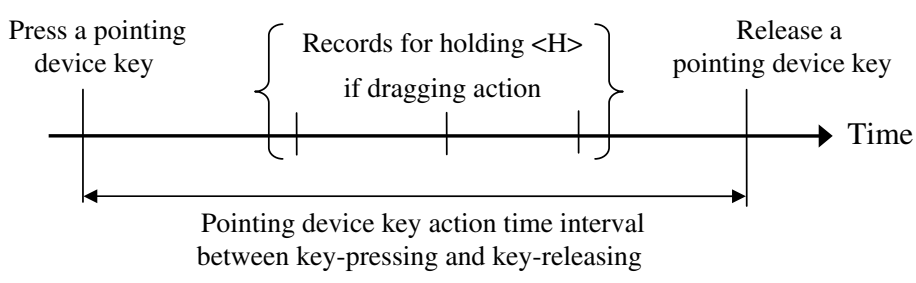

Illustration of time interval calculations for clicking and dragging actions.

Fig. 1. Examples and illustration of Mlog monitoring program logging system and time interval calculations for clicking, dragging, and moving actions.

pressed and released the pointing device key to collect pairs of time intervals recorded by the oscilloscope and the Mlog monitoring program, continuing until 10 time intervals monitored by the oscilloscope were exactly equal to the specified time interval level. Then, the 10 corresponding time intervals recorded by the Mlog monitoring program were compared to the specified time interval recorded by the oscilloscope in order to evaluate the accuracy and precision of the former. Ten measurements were chosen here because, in the pilot study, the relative standard deviations for 10 measurements by the oscilloscope or Mlog monitoring program, for pointing device action time intervals of $200 \sim 1000 \mathrm{~ms}$, were $0.42 \sim 1.47 \%$; these were thought precise enough in this part of the evaluation. Mlog accuracy and precision were calculated as follows:

$$
\begin{gathered}
\operatorname{Accuracy}(\%)=[\text { (Mlog recording }- \text { tested time interval }) \\
\div(\text { tested time interval })] \times 100 \\
\text { Precision error }(\%)=[(\text { standard deviation of Mlog recording }) \\
\div(\text { mean Mlog recording })] \times 100
\end{gathered}
$$

Reliability testing for the Mlog monitoring program was performed based on various computer sets, computer system loadings,

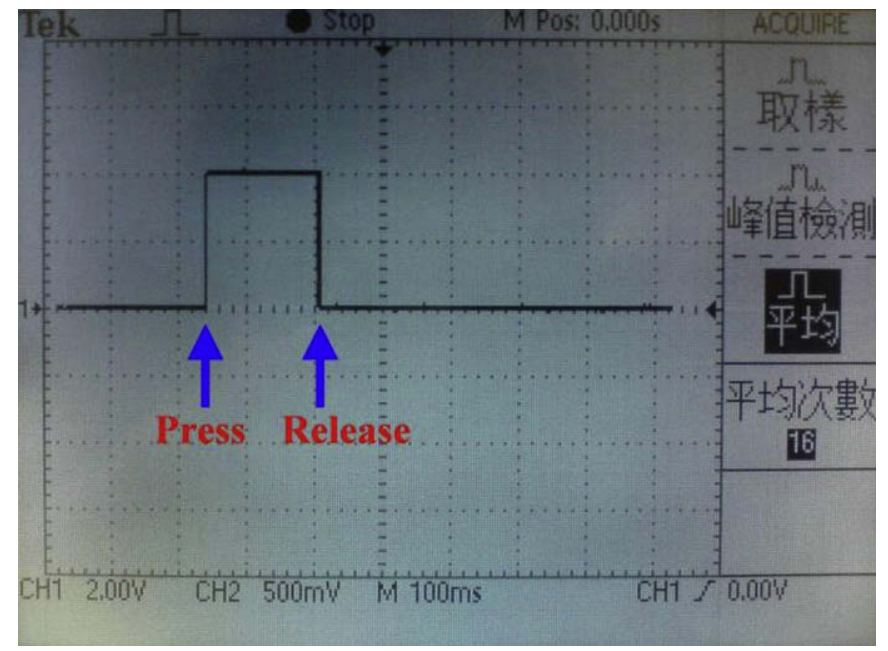

Fig. 2. Example of screen capture from oscilloscope for the recorded key pressing and releasing times of clicking action of the pointing device. 
and time intervals for pointing device actions. Computer sets were sorted into three groups based on differences in CPU speed and memory capacity, i.e., $0.993 \mathrm{GHz}$ and $128 \mathrm{MB}, 2.02 \mathrm{GHz}$ and $256 \mathrm{MB}$, and $3.20 \mathrm{GHz}$ and $990 \mathrm{MB}$. Computer system loadings common in the workplace were simulated and classified into 4 levels as described below:

I. Minimum: only Mlog was run, representing minimum system loading for the computer. An average of around $2 \%$ of CPU used, with infrequent use up to $5 \%$.

II. Light: only Mlog and Microsoft Word were run, representing light system loading. An average of around 7\% CPU usage, with infrequent use up to $12 \%$.

III. Medium: in addition to Mlog, five software programs, specifically Microsoft Word, Excel, Access, Outlook and Explore, were simultaneously run on the computer system. An average of around $12 \%$ CPU was used, with infrequent use up to $60 \%$.

IV. Heavy: in addition to those software programs run in the medium loading condition, additional software programs, including Media Player, Acrobat Reader, SAS, KKman and PCman, were also run to simulate a heavy load on system resources (hard disc, memory capacity, and CPU). An average of around 30\% CPU usage, with infrequent use up to $90 \%$.

Time intervals of pointing device actions for the test were grouped into 8 levels based on the oscilloscope records: $75 \sim 125$ $\mathrm{ms}, \quad 175 \sim 225 \mathrm{~ms}, \quad 250 \sim 350 \mathrm{~ms}, \quad 400 \sim 500 \mathrm{~ms}, 800 \sim 1,200 \mathrm{~ms}$, $1800 \sim 2200 \mathrm{~ms}, \quad 2800 \sim 3200 \mathrm{~ms}$ and $9800 \sim 10,200 \mathrm{~ms}$. These covered the time intervals ranging from those for clicking action to those for dragging and/or moving actions.

In the reliability testing for the Mlog monitoring program, for each combination of specific computer set, computer system loading, and time interval group, pointing device activity was repetitively monitored until there were 20 time interval measurements within the specified range of the time interval group. The time intervals obtained by the oscilloscope were used as references to evaluate the reliability of the recording logged by the Mlog monitoring program, where these were evaluated as follows in terms of deviation, relative error, and ratio:

$\operatorname{Deviation}(\mathrm{ms})=($ time interval of pointing device action recorded by Mlog monitoring program) - (time interval of pointing device action recorded by oscilloscope).

Relative error $(\%)=\mid($ time interval of pointing device action recorded by Mlog monitoring program) - (time interval of pointing device action recorded by oscilloscope) $\mid \div$ (time interval of pointing device action recorded by oscilloscope).

Ratio $=$ (time interval of pointing device action recorded by Mlog monitoring program $) \div$ (time interval of pointing device action recorded by oscilloscope).

To reduce the size of the data file for pointing device moving action monitoring, the Mlog system allows users to select different sampling rates - i.e., 7.1, 8.0, 9.2, 10.7, 12.8, 16.0, 21.4, 32.1, or $64.1 \mathrm{samples} / \mathrm{s}$ - based on the signal transmission interval of $15.6 \mathrm{~ms}$ for the computer used in the present study. The accuracy for pointing device moving action time interval monitoring was also evaluated at different sampling rates by examining the deviation and relative error of data recorded by the Mlog monitoring program as compared to those recorded by the oscilloscope on a computer with minimum computer system loading.

\subsection{Statistical analysis}

For the evaluation of accuracy and precision, each 10 time intervals recorded by the Mlog monitoring program were examined by comparing them to the specifically designated tested time intervals with $t$-test. Meanwhile, accuracy and precision for Mlog recordings were also presented using percentage.

Data points collected from all tested time interval groups for reliability testing by the Mlog monitoring program were put together for statistical analysis. Univariate analyses were conducted for deviation, relative error, and the ratio of pointing device action time intervals recorded by the Mlog monitoring program, as compared to those recorded by the oscilloscope; this enabled us to describe the reliability of the recorded Mlog data by computer set, computer system loading and tested time interval group. Meanwhile, the deviation of pointing device action time intervals recorded by the Mlog monitoring program was also examined using paired $t$-test comparing them to those recorded by the oscilloscope, with the significance level set at 0.05 .

Furthermore, correlation analysis and intraclass correlation analysis (ICC) were conducted between the pointing device action time intervals recorded by the Mlog monitoring program and those recorded by the oscilloscope. These were also organized by computer set, computer system loading and pointing device action. An analysis of variance (ANOVA) was also conducted to compare the effects of computer set, computer system loading, and pointing device key action on the correlation coefficients for pointing device action time intervals recorded by the Mlog monitoring program and the oscilloscope. Multiple linear regression analysis was applied to examine the effects of computer set, computer system loading, pointing device action, and length of tested time interval on the Mlog monitoring records, and to determine how much of the variance in time interval recording was explained by the regression model $\left(r^{2}\right)$. In addition to the predictor of tested time interval level, other predictors were added to the regression model singly or simultaneously in different trials to examine their individual effects. All statistical analyses were carried out using Statistical Analysis System 9.1.3 (SAS Institute Inc., Cary, NC, USA).

\section{Results}

The time interval recordings made by the Mlog monitoring program were not statistically different from the corresponding tested time intervals, except for the tested time intervals of $200 \mathrm{~ms}$ and $1000 \mathrm{~ms}(p<0.05)$ (Table 1$)$. In general, measurement errors for the Mlog monitoring program were within $\pm 0.50 \%$, except for the tested time intervals of 100 and $200 \mathrm{~ms}$ (Table 1). Precision error was less than $1.00 \%$ for the tested time intervals equal to or greater than $450 \mathrm{~ms}$, while it was less than $3.00 \%$ for the other three shorter tested time intervals.

Results of reliability testing show that, for the tested time interval groups for pointing device clicking action $(<500 \mathrm{~ms})$, most differences between pointing device action time intervals recorded by the Mlog monitoring program and those recorded by the oscilloscope were within $\pm 1 \mathrm{~ms}$, whereas those for the tested time interval groups for pointing device dragging action ( $>800 \mathrm{~ms}$ ) were mostly within $\pm 3 \mathrm{~ms}$. Among all tested time interval groups, some recordings by Mlog monitoring program were slightly different from the references obtained using the oscilloscope $(p<0.05)$, including tested time interval groups of $175 \sim 225 \mathrm{~ms}$ and $400 \sim 500 \mathrm{~ms}$ for computer set C, $250 \sim 350 \mathrm{~ms}$ for computer set B, and $1800 \sim 2200 \mathrm{~ms}$ for computer set A. For 
Table 1

Accuracy and precision error of pointing device action time interval recorded by Mlog monitoring program.

\begin{tabular}{lllll}
\hline $\begin{array}{l}\text { Tested time } \\
\text { interval, }{ }^{\mathrm{a}} \mathrm{ms}\end{array}$ & $\begin{array}{l}\text { Sample size } \\
\text { ( }\end{array}$ & $\begin{array}{l}\text { Time interval } \\
\text { recorded } \\
\text { by Mlog, } \mathrm{ms}\end{array}$ & $\begin{array}{l}\text { Accuracy error, } \\
\%\end{array}$ & $\begin{array}{l}\text { Precision error, } \\
\%\end{array}$ \\
\hline 100 & 10 & $100.80 \pm 2.86$ & $0.80 \pm 2.86$ & 2.84 \\
200 & 10 & $197.60 \pm 2.91^{*}$ & $-1.20 \pm 1.46$ & 1.47 \\
300 & 10 & $301.50 \pm 4.99$ & $0.50 \pm 1.66$ & 1.66 \\
450 & 10 & $451.40 \pm 4.30$ & $0.31 \pm 0.96$ & 0.95 \\
1000 & 10 & $1003.30 \pm 4.16^{*}$ & $0.33 \pm 0.42$ & 0.42 \\
2000 & 10 & $1996.50 \pm 19.20$ & $-0.18 \pm 0.96$ & 0.96 \\
3000 & 10 & $3008.30 \pm 15.83$ & $0.28 \pm 0.53$ & 0.53 \\
10,000 & 10 & $10,008.60 \pm 66.09$ & $0.09 \pm 0.66$ & 0.66 \\
\hline
\end{tabular}

${ }^{*} p<0.05, t$-test compared to the tested time interval.

a Tested time interval: time interval recorded by oscilloscope.

most tested time interval groups, the relative error for pointing device action time intervals recorded by the Mlog monitoring program was less than $1 \%$, with the exception of the time interval groups of $75 \sim 125 \mathrm{~ms}$ and $175 \sim 225 \mathrm{~ms}$, for which the maximum relative errors were $2.81 \%$ and $1.44 \%$, respectively. For the ratio of pointing device action time interval recorded by the Mlog monitoring program to those calculated by oscilloscope, results show that these ratios ranged from $0.9964 \pm 0.0113$ to $1.0038 \pm 0.0154$, varying by the tested time interval. As the tested time interval became longer, the ratios got closer to 1.0000 , with narrower standard deviation.

Pointing device action time intervals recorded by the Mlog monitoring program were highly correlated with those by oscilloscope, with correlation coefficients ranging from 0.99905 to $0.99999(p<0.0001)$. Although all these coefficients were very high, the results of the analysis of variance (ANOVA) still indicated higher correlation coefficients for the tested time intervals of pointing device dragging action, as compared to those of clicking action $(p<0.0001)$. However, no obvious trend was found for computer set and computer system loading. Meanwhile, intraclass correlation analysis showed that high coefficients were observed for all tested time interval groups (Table 2). Intraclass correlation coefficients for the tested time interval groups of clicking actions ( $\leqq 400 \sim 500 \mathrm{~ms}$ ) were mostly greater than 0.95 , whereas a few dragging actions with longer tested time intervals $2800 \sim 3200 \mathrm{~ms}$ and $9600 \sim 10,400 \mathrm{~ms}$ - were characterized with coefficients less than 0.90 .

The results of the multiple regression analyses in Table 4 indicate that the tested time interval level was the only significant predictor of time interval recordings made by the Mlog monitoring program $(p<0.0001)$ and dominated the explained variance $\left(r^{2}=0.9999\right)$. No effect was observed to be due to differences in computer set, computer system loading, or type of pointing device action, no matter whether these predictors were included in the model separately or simultaneously.

For the influence of record-sampling rates for pointing device movement monitoring, results of the accuracy test shown in Fig. 3 indicate that the deviation of data recorded by the Mlog monitoring program from those recorded using the oscilloscope ranged from -0.43 to $0.02 \mathrm{~ms}$, with relative errors ranging from $0.87 \%$ to $1.33 \%$.

\section{Discussion}

Electronic activity monitoring methods that on-line direct the electronic signals from the keyboard and pointing device to the

Table 2

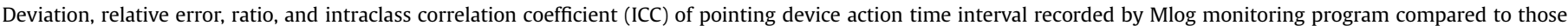
recorded by oscilloscope by tested time interval group.

\begin{tabular}{|c|c|c|c|c|c|c|}
\hline Computer set & Pointing device action & Tested time interval group & Deviation & Relative error, \% & Ratio & ICC (95\%CI) \\
\hline \multirow[t]{8}{*}{$\bar{A}$} & Clicking & $75 \sim 125 \mathrm{~ms}$ & $0.30 \pm 3.30$ & $2.58 \pm 2.00$ & $1.0030 \pm 0.0326$ & $0.92(0.88,0.95)$ \\
\hline & & $175 \sim 225 \mathrm{~ms}$ & $0.33 \pm 3.56$ & $1.44 \pm 1.04$ & $1.0015 \pm 0.0178$ & $0.95(0.92,0.97)$ \\
\hline & & $250 \sim 350 \mathrm{~ms}$ & $0.21 \pm 3.08$ & $0.84 \pm 0.57$ & $1.0007 \pm 0.0101$ & $0.96(0.94,0.97)$ \\
\hline & & $400 \sim 500 \mathrm{~ms}$ & $0.55 \pm 5.10$ & $0.91 \pm 0.67$ & $1.0012 \pm 0.0113$ & $0.97(0.95,0.98)$ \\
\hline & Dragging & $800 \sim 1200 \mathrm{~ms}$ & $0.54 \pm 5.91$ & $0.49 \pm 0.32$ & $1.0006 \pm 0.0058$ & $0.97(0.95,0.98)$ \\
\hline & & $1800 \sim 2200 \mathrm{~ms}$ & $-4.70 \pm 15.89^{*}$ & $0.67 \pm 0.47$ & $0.9977 \pm 0.0079$ & $0.94(0.91,0.96)$ \\
\hline & & $2800 \sim 3200 \mathrm{~ms}$ & $1.11 \pm 24.62$ & $0.54 \pm 0.62$ & $1.0004 \pm 0.0082$ & $0.89(0.83,0.93)$ \\
\hline & & $9600 \sim 10,400 \mathrm{~ms}$ & $8.36 \pm 49.03$ & $0.41 \pm 0.28$ & $1.0009 \pm 0.0049$ & $0.88(0.82,0.92)$ \\
\hline \multirow[t]{8}{*}{ B } & Clicking & $75 \sim 125 \mathrm{~ms}$ & $0.28 \pm 3.73$ & $2.81 \pm 2.46$ & $1.0032 \pm 0.0374$ & $0.94(0.91,0.96)$ \\
\hline & & $175 \sim 225 \mathrm{~ms}$ & $0.54 \pm 5.01$ & $1.40 \pm 1.98$ & $1.0024 \pm 0.0242$ & $0.93(0.89,0.95)$ \\
\hline & & $250 \sim 350 \mathrm{~ms}$ & $-1.09 \pm 3.46^{*}$ & $0.90 \pm 0.76$ & $0.9964 \pm 0.0113$ & $0.97(0.95,0.98)$ \\
\hline & & $400 \sim 500 \mathrm{~ms}$ & $0.28 \pm 5.15$ & $0.88 \pm 0.70$ & $1.0008 \pm 0.0113$ & $0.97(0.95,0.98)$ \\
\hline & Dragging & $800 \sim 1200 \mathrm{~ms}$ & $0.25 \pm 7.97$ & $0.63 \pm 0.46$ & $1.0003 \pm 0.0079$ & $0.96(0.94,0.97)$ \\
\hline & & $1800 \sim 2200 \mathrm{~ms}$ & $0.19 \pm 15.27$ & $0.60 \pm 0.46$ & $1.0001 \pm 0.0076$ & $0.96(0.94,0.97)$ \\
\hline & & $2800 \sim 3200 \mathrm{~ms}$ & $-2.46 \pm 17.50$ & $0.49 \pm 0.32$ & $0.9992 \pm 0.0058$ & $0.94(0.91,0.96)$ \\
\hline & & $9600 \sim 10,400 \mathrm{~ms}$ & $0.74 \pm 73.77$ & $0.59 \pm 0.43$ & $1.0001 \pm 0.0073$ & $0.85(0.78,0.90)$ \\
\hline \multirow[t]{8}{*}{$\bar{C}$} & Clicking & $75 \sim 125 \mathrm{~ms}$ & $0.25 \pm 3.23$ & $2.35 \pm 1.97$ & $1.0027 \pm 0.0307$ & $0.95(0.92,0.97)$ \\
\hline & & $175 \sim 225 \mathrm{~ms}$ & $0.76 \pm 3.07^{*}$ & $1.27 \pm 0.93$ & $1.0038 \pm 0.0154$ & $0.97(0.95,0.98)$ \\
\hline & & $250 \sim 350 \mathrm{~ms}$ & $0.34 \pm 3.62$ & $0.87 \pm 0.83$ & $1.0010 \pm 0.0120$ & $0.97(0.95,0.98)$ \\
\hline & & $400 \sim 500 \mathrm{~ms}$ & $1.45 \pm 4.92^{*}$ & $0.86 \pm 0.75$ & $1.0033 \pm 0.0110$ & $0.98(0.97,0.99)$ \\
\hline & Dragging & $800 \sim 1200 \mathrm{~ms}$ & $0.15 \pm 6.67$ & $0.52 \pm 0.41$ & $1.0002 \pm 0.0066$ & $0.99(0.98,0.99)$ \\
\hline & & $1800 \sim 2200 \mathrm{~ms}$ & $-1.91 \pm 16.19$ & $0.60 \pm 0.53$ & $0.9991 \pm 0.0080$ & $0.95(0.92,0.97)$ \\
\hline & & $2800 \sim 3200 \mathrm{~ms}$ & $2.25 \pm 20.40$ & $0.48 \pm 0.47$ & $1.0008 \pm 0.0067$ & $0.91(0.86,0.94)$ \\
\hline & & $9600 \sim 10,400 \mathrm{~ms}$ & $-2.74 \pm 59.40$ & $0.46 \pm 0.37$ & $0.9998 \pm 0.0059$ & $0.86(0.79,0.91)$ \\
\hline \multirow[t]{8}{*}{$\overline{\text { Total }}$} & Clicking & $75 \sim 125 \mathrm{~ms}$ & $0.28 \pm 3.41$ & $2.58 \pm 2.16$ & $1.0030 \pm 0.0335$ & $0.94(0.92,0.95)$ \\
\hline & & $175 \sim 225 \mathrm{~ms}$ & $0.54 \pm 3.95^{*}$ & $1.37 \pm 1.40$ & $1.0025 \pm 0.0194$ & $0.95(0.94,0.96)$ \\
\hline & & $250 \sim 350 \mathrm{~ms}$ & $-0.18 \pm 3.44$ & $0.87 \pm 0.73$ & $0.9994 \pm 0.0113$ & $0.97(0.96,0.98)$ \\
\hline & & $400 \sim 500 \mathrm{~ms}$ & $0.76 \pm 5.06$ & $0.88 \pm 0.70$ & $1.0018 \pm 0.0112$ & $0.97(0.96,0.98)$ \\
\hline & Dragging & $800 \sim 1200 \mathrm{~ms}$ & $0.31 \pm 6.88$ & $0.55 \pm 0.41$ & $1.0003 \pm 0.0068$ & $0.98(0.97,0.98)$ \\
\hline & & $1800 \sim 2200 \mathrm{~ms}$ & $-2.14 \pm 15.85^{*}$ & $0.62 \pm 0.49$ & $0.9989 \pm 0.0079$ & $0.95(0.94,0.96)$ \\
\hline & & $2800 \sim 3200 \mathrm{~ms}$ & $0.30 \pm 21.05$ & $0.50 \pm 0.48$ & $1.0001 \pm 0.0070$ & $0.91(0.89,0.93)$ \\
\hline & & $9600 \sim 10,400 \mathrm{~ms}$ & $2.12 \pm 61.49$ & $0.49 \pm 0.37$ & $1.0002 \pm 0.0061$ & $0.86(0.82,0.89)$ \\
\hline
\end{tabular}

${ }^{*} p<0.05$, Paired $t$-test between time intervals of pointing device action recorded by Mlog monitoring program and oscilloscope, respectively. 
designated computer recording system have the advantage of seeing almost no effect of computer memory capacity and computer system loadings on the accuracy of monitored records (Homan and Armstrong, 2003; Johnson et al., 2000). On the other hand, for the Mlog monitoring software program, the recorded time is based on the high-resolution timer in kernel mode, rather than on the system timer in the user mode, for the computer with a resolution of $0.28 \mathrm{~ms}$. Since the data transmission path is very complicated, the recorded time data are first saved and then retrieved for time calculation. Therefore, these temporarily saved data may consistently occupy parts of the CPU, and data retrieval may experience a time lag (Russinovich and Solomon, 2004). Nevertheless, since data retrieval from the kernel mode is intermittently conducted when the CPU is not busy, the execution of the Mlog monitoring program in this study, performed on different computer sets with different computer system loadings, usually occupied less than $2 \%$ of the CPU resources and infrequently occupied up to $5 \%$.

On the other hand, as shown in the evaluation of accuracy and precision for the Mlog monitoring program, it was noted that both accuracy and precision improved as the tested time interval increased from $100 \mathrm{~ms}$ to $10,000 \mathrm{~ms}$ (Table 1). Although the recording difference in the time interval by Mlog might have increased as the interval time increased, obviously decreasing trends were observed for accuracy error, decreasing from about $1.0 \%$ to $0.1 \%$, and precision error, decreasing from $2.8 \%$ to $0.6 \%$. A similar trend was also observed in the relative error for the Mlog monitoring program with different computer conditions in operation (Table 2). For the pointing device dragging action, with relatively longer time intervals of $800 \sim 12,000 \mathrm{~ms}$, the relative Mlog recording errors were as low as about $0.5 \%$, whereas the relative errors for clicking actions decreased from about $2.5 \%$ for the tested time interval group of $75 \sim 125 \mathrm{~ms}$ to about $0.9 \%$ for that of $400 \sim 500 \mathrm{~ms}$ (Table 2 ).

With respect to shorter tested time interval groups, although the relative errors by the Mlog monitoring program were generally higher than those of longer tested time interval groups, their average deviations from the corresponding references were relatively small and less than $1 \mathrm{~ms}$ (Table 2 ). It has been indicated that, with a single and simple stimulus, human reaction time is around $150 \sim 200 \mathrm{~ms}$ if warning occurs $1 \mathrm{~s}$ before the stimulus (Hsiao, 2007). For instance, the time interval between pressing and releasing a keypad, when one is typing on a computer keyboard, is about $100 \sim 200 \mathrm{~ms}$ (Chang et al., 2004). Compared to these human reaction time levels, the deviations for clicking action time intervals monitored by the Mlog monitoring program - i.e., $0.28 \pm 3.41 \mathrm{~ms}$ for the tested time interval group of $75 \sim 125 \mathrm{~ms}$, and $0.54 \pm 3.95 \mathrm{~ms}$ for that of $175 \sim 225 \mathrm{~ms}$ (Table 2) - can be

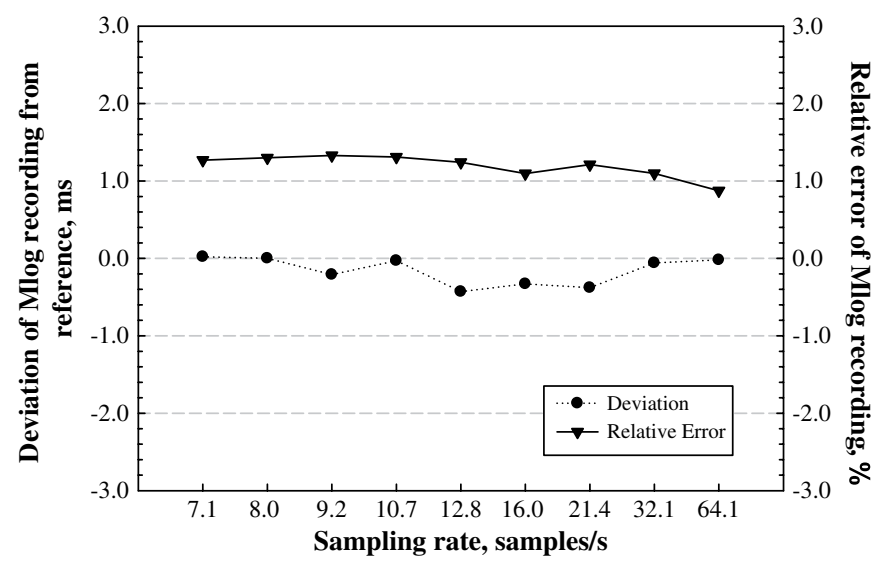

Fig. 3. Accuracy and relative error of the pointing device moving action monitoring by Mlog as compared to the references by oscilloscope. considered small and acceptable because they do not present significant bias in the pointing device action monitoring.

Additionally, correlation coefficients between the Mlog recordings and the references were very high and all greater than 0.99905 (Table 3). Among them, those for the dragging actions were significantly higher than those for the clicking actions were. Meanwhile, further analysis of the reliability test also indicated high intraclass correlation coefficients between Mlog recordings and the references (Table 2). Furthermore, the findings of this study indicated that the pointing device action monitored by the Mlog program was hardly affected by computer sets and computer system loadings in ordinary use (Tables 3 and 4). In general, although the Mlog monitoring process might delay the absolute time record to some extent, the relative time difference was much less affected based on the aforementioned supporting evidences. A validity test of the Mlog monitoring software program indicated that this program is reliable in the monitoring of pointing device activity during the computer work.

Electronic activity records collected by the Mlog monitoring program can be used to estimate computer pointing device use time during a computer task. If Mlog works together with an electronic activity monitoring program previously developed by the authors' laboratory for computer keyboard activity monitoring (Chang et al., 2004), these two monitoring programs may jointly estimate total computer use time, keyboard use time, and mouse use time. In recent years, certain kinds of electronic activity monitoring were also available through computer usage software programs with similar functions in terms of monitoring computer electronic activity during computer tasks - for example, a commercial software package (Workpace, Niche Software Ltd., New Zealand), and others developed by individual research laboratories (Dennerlein and Johnson, 2006; Homan and Armstrong, 2003). These electronic activity monitoring programs have been used to evaluate the effects of different computer tasks on the exposure of the upper extremities to biomechanical risk factors (Dennerlein and Johnson, 2006), to

Table 3

Correlation coefficients between pointing device action time intervals recorded by Mlog monitoring program and oscilloscope by computer set, computer system loading and pointing device key action. ${ }^{c}$

\begin{tabular}{|c|c|c|c|c|}
\hline \multirow[t]{2}{*}{ Computer set $^{\mathrm{a}}$} & \multirow[t]{2}{*}{ Computer system loading b } & \multicolumn{2}{|c|}{$\begin{array}{l}\text { Pointing device } \\
\text { action }\end{array}$} & \multirow[t]{2}{*}{ Total } \\
\hline & & Clicking & Dragging & \\
\hline $\bar{A}$ & $\begin{array}{l}\text { I } \\
\text { II } \\
\text { III } \\
\text { VI } \\
\text { Subtotal }\end{array}$ & $\begin{array}{l}0.99958 \\
0.99964 \\
0.99947 \\
0.99962 \\
0.99958\end{array}$ & $\begin{array}{l}0.99997 \\
0.99998 \\
0.99998 \\
0.99995 \\
0.99997\end{array}$ & $\begin{array}{l}0.99998 \\
0.99999 \\
0.99998 \\
0.99997 \\
0.99998\end{array}$ \\
\hline B & $\begin{array}{l}\text { I } \\
\text { II } \\
\text { III } \\
\text { VI } \\
\text { Subtotal }\end{array}$ & $\begin{array}{l}0.99905 \\
0.99958 \\
0.99956 \\
0.99962 \\
0.99945\end{array}$ & $\begin{array}{l}0.99994 \\
0.99994 \\
0.99995 \\
0.99994 \\
0.99994\end{array}$ & $\begin{array}{l}0.99996 \\
0.99996 \\
0.99996 \\
0.99996 \\
0.99996\end{array}$ \\
\hline C & $\begin{array}{l}\text { I } \\
\text { II } \\
\text { III } \\
\text { VI } \\
\text { Subtotal }\end{array}$ & $\begin{array}{l}0.99981 \\
0.99973 \\
0.99943 \\
0.99946 \\
0.99958\end{array}$ & $\begin{array}{l}0.99992 \\
0.99997 \\
0.99997 \\
0.99998 \\
0.99996\end{array}$ & $\begin{array}{l}0.99995 \\
0.99998 \\
0.99998 \\
0.99998 \\
0.99997\end{array}$ \\
\hline Total & $\begin{array}{l}\text { I } \\
\text { II } \\
\text { III } \\
\text { VI } \\
\text { Subtotal }\end{array}$ & $\begin{array}{l}0.99946 \\
0.99965 \\
0.99949 \\
0.99953 \\
0.99953\end{array}$ & $\begin{array}{l}0.99994 \\
0.99996 \\
0.99996 \\
0.99996 \\
0.99996\end{array}$ & $\begin{array}{l}0.99996 \\
0.99998 \\
0.99997 \\
0.99997 \\
0.99997\end{array}$ \\
\hline
\end{tabular}

\footnotetext{
${ }^{a}$ Computer set: A - $0.933 \mathrm{GHz}, 128 \mathrm{MB}$; B - $2.02 \mathrm{GHz}, 256 \mathrm{MB}$; C $-3.20 \mathrm{GHz}$ $990 \mathrm{MB}$.

b Computer system loading: I - Minimum; II - Light; III - Medium; IV - Heavy.
}

c All correlation coefficients are statistically significant with $p<0.0001$. 


\section{Table 4}

Linear regression models for the effects of tested time interval (oscilloscope recording), computer set, computer system loading, and pointing device key action on the pointing device action time interval recorded by Mlog monitoring program. ${ }^{\text {a }}$

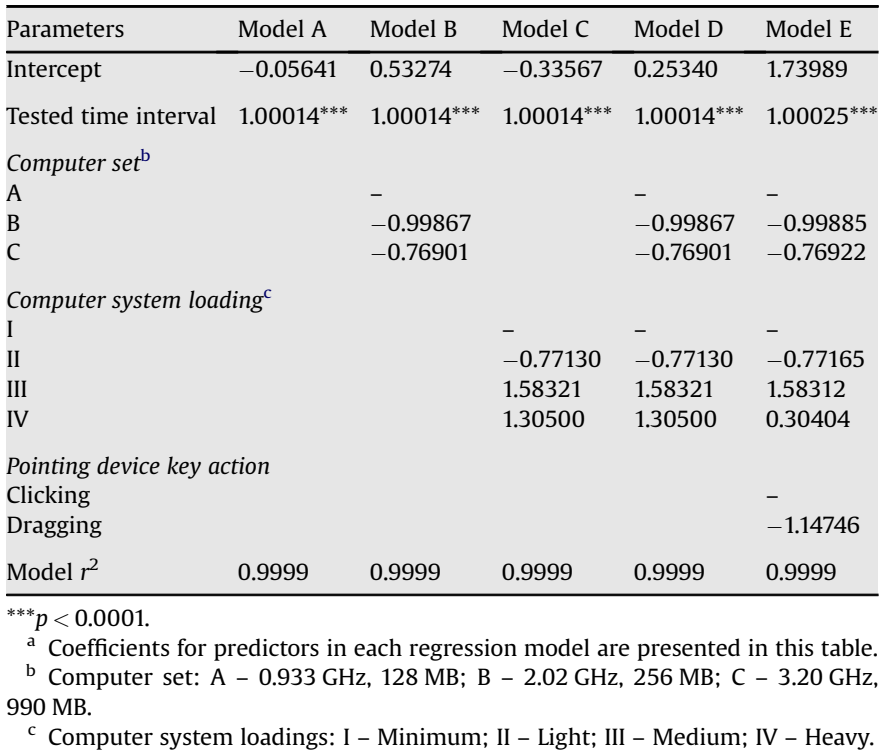

predict acute pain in the neck and shoulder for computer mouse use (Andersen et al., 2008), to validate the questionnaire self-reports on computer, mouse and keyboard usage during a four-week period (Mikkelsen et al., 2007), and to evaluate self-reported systems with temporal change in bimanual inter-key press intervals during continuous typing (Liang et al., 2008). These wide applications reflect one of the advantages of electronic activity monitoring, that of using standardized indicators derived from the collected data for computer activity monitoring to compare physical work loadings for a wide variety of computer tasks.

Furthermore, future study is merited that would explore new indices derived from the electronic activity records collected by the Mlog monitoring program to assess computer users' work load with computer tasks: for example, key holding time for clicking actions between pressing and releasing a key, or the speed and/or acceleration of pointing device moving action. For computer work load assessments, many studies find evidence of reduced task performance after 40-60 min of typing or data entry, but the cause has not been well documented (Floru et al., 1985; Gerard et al., 1996; Lin et al., 2004). However, since computer work involves only low-force repetitions of hand/finger movements, it is likely that conventional instruments for muscular fatigue measurement, such as surface electromyography (sEMG), may not be sensitive enough to detect such muscular fatigue, if any exists (Gerard et al., 1996; Lin et al., 2004). In contrast, the Mlog monitoring program is able to provide continuous real-time monitoring records and self-reference for individual comparison, to reflect subtle changes in muscular performance in terms of key holding time for clicking action or speed and/or acceleration for moving action. This prompts the possibility for these newly proposed measures to be used as indirect indicators of such muscular fatigue. Of course, further study is accordingly necessary in order to examine and validate these indicators as alternatives for muscular fatigue assessment, and in order to elaborate their relationship to task performance in computer work.

Although Mlog is convenient and time-saving for computer activity monitoring, and causes almost no interference to the subject being monitored, it carries the same limitation that all types of electronic activity monitoring methods do: i.e., it measures only the computer use times when the input device is activated. In this case, the calculation of pointing device use time is based on the duration of the interval between clicking actions and/or moving actions or vice versa, according to a pre-defined idle time. If the interval is shorter than the idle time, it is recorded as time spent using the computer pointing device, while an interval of longer duration is recorded as a break. For electronic activity monitoring, the threshold set for idle time is critical in the computer use time estimation. Different idle time thresholds of $2 \mathrm{~s}$ and $5 \mathrm{~s}$ have ever been applied in previous studies to calculate the computer mouse use time, and to differentiate use time from non-use time during the monitoring period (Mikkelsen et al., 2007; Dennerlein and Johnson, 2006; Homan and Armstrong, 2003). However, with the application of idle time thresholds, it is still possible to misclassify the registration for computer use times. For instance, electronic activity monitoring does not include the time taken up by passive computer tasks, like viewing the computer screen without activating any pointing device. This may confound the exposure measurement of pointing device use time through electronic activity monitoring. Therefore, as one type of electronic activity monitoring, Mlog must be used cautiously in the estimation of pointing device use times for computer tasks. It is recommended that this monitoring tool only be used in estimations for intensive pointing device activity along with limited passive computer use time. Further study is warranted to establish criteria for passive computer use time in proportion to total computer use time, in order to decide how small the proportion of passive computer use time should be in order for one to appropriately use Mlog for computer pointing device monitoring.

Job type is another concern with the electronic activity monitoring. Variety in job type may influence the computer use time estimation. It was reported that the activity for some jobs, like that of computer experts, might be underestimated by electronic activity monitoring - whereas others might tend to be overestimated (Douwes et al., 2007). On the other hand, a previous study further indicated that typing was predominantly a keyboard task, that the sorting and resizing of graphical objects and intranet web page browsing were predominantly mouse tasks, and that the HTML-based formatting and text editing tasks used a mixture of the two devices (Dennerlein and Johnson, 2006). Therefore, further study is also needed to explore if there is any potential effect of job type on the performance of Mlog monitoring in field studies for computer pointing device activity.

In conclusion, with limited estimate deviation and high reliability as compared to the references, the Mlog monitoring program was validated as accurate in measuring pointing device activity during computer work. In addition to being used to calculate pointing device use time estimations, these data collected by the Mlog monitoring program may also possess the potential to be further explored as an alternative indicator for computer users' hand muscular performance or even muscular fatigue. However, the Mlog monitoring program does not monitor actual hand movements, postures; therefore, it could only be thought of as providing indirect and supplemental exposure indices for those traditional ergonomic measurements for physical loading assessment. Additionally, special attention should be paid to the confounding effect of passive computer activity on the use time estimation made by the Mlog monitoring program. Future study is also warranted to examine the influences of idle time, and job type on the performance of the Mlog monitoring program in field studies.

\section{References}

Allison, G.T., 2003. Trunk muscle onset detection technique for EMG signals with ECG artefact. Journal of Electromyography and Kinesiology 13, 209-216.

Andersen, J.H., Harhoff, M., Grimstrup, S., Vilstrup, I., Lassen, C.F., Brandt, L.P.A., et al., 2008. Computer mouse use predicts acute pain but not prolonged or chronic pain in the neck and shoulder. Occupational and Environmental Medicine 65,126-131. 
Bergdahl, J., Stenberg, B., Eriksson, N., Linden, G., Widman, L., 2004. Coping and selfimage in patients with visual display terminal-related skin symptoms and perceived hypersensitivity to electricity. International Archives of Occupational and Environmental Health 77 (8), 538-542.

Bergqvist, U., Wolgast, E., Nilsson, B., Voss, M., 1995. Musculoskeletal disorders among visual display terminal workers: individual, ergonomic, and work organizational factors. Ergonomics 38, 763-776.

Blehm, C., Vishnu, S., Khattak, A., Mitra, S., Yee, R.W., 2005. Computer vision syndrome: a review. Survey of Ophthalmology 50 (3), 253-262.

Chang, C.H., Wang, J.D., Luh, J.J., Hwang, Y.H., 2004. Development of a monitoring system for keyboard users' performance. Ergonomics 47 (14), 1571-1581.

Dennerlein, J.T., Johnson, P.W., 2006. Different computer tasks affect the exposure of the upper extremity to biomechanical risk factors. Ergonomics 49 (1), 45-61.

Douwes, M., de Kraker, H., Blatter, B.M., 2007. Validity of two methods to assess computer use: self-report by questionnaire and computer use software. International Journal Industrial Ergonomics 37, 425-431.

Faucett, J., Rempel, D., 1994. VDT-related musculoskeletal symptoms: interactions between work posture and psychosocial work factors. American Journal of Industrial Medicine 26, 597-612.

Floru, R., Cail, F., Elias, R., 1985. Psychophysiological changes during a VDU repetitive task. Ergonomics 28, 1455-1468.

Gerard, M.J., Armstrong, T.J., Foulke, J.A., Martin, B.J., 1996. Effects of key stiffness on force and the development of fatigue while typing. American Industrial Hygiene Association Journal 57, 849-854.

Homan, M.M., Armstrong, T.J., 2003. Evaluation of three methodologies for assessing work activity during computer use. American Industrial Hygiene Association Journal 64, 48-55.

Hsiao, H.W., 2007. Occupational safety and human factors. In: Rom, W.N., Markowitz, S.B. (Eds.), Environmental and Occupational Medicine, fourth ed. Wolters Kluwer/Lippincott Williams \& Wilkins, Philadelphia.

Jensen, C., Finsen, L., Hansen, K., Christensen, H., 1999. Upper trapezius muscle activity patterns during repetitive manual material handling and work with a computer mouse. Journal of Electromyography and Kinesiology 9, 317-325.

Johnson, P.W., Hagberg, M., Hjelm, E.W., Rempel, D., 2000. Measuring and characterizing force exposures during computer mouse use. Scandinavian Journal of Work, Environment and Health 26 (5), 398-405.

Jonsson, P., Johnson, P.W., Hagberg, M., 2007. Accuracy and feasibility of using an electrogoniometer for measuring simple thumb movements. Ergonomics 50 (5), 647-659.

Karlqvist, L.K., Bernmark, E., Ekenvall, L., Hagberg, M., Isaksson, A., Rosto, T., 1998. Computer mouse position as a determinant of posture, muscular load and perceived exertion. Scandinavian Journal of Work, Environment and Health 24 (1), 62-73.

Keir, P.J., Bach, J.M., Rempel, D., 1999. Effects of computer mouse design and task on carpal tunnel pressure. Ergonomics 42, 1350-1360.

Kimura, M., Sato, H., Ochi, M., Hosoya, S., Sadoyama, T., 2007. Electromyogram and perceived fatigue changes in the trapezius muscle during typewriting and recovery. European Journal of Applied Physiology 100, 89-96.
Koren, G., 2003. Exposure to electromagnetic fields during pregnancy. Canadian Family Physician 49, 151-153.

Kotani, K., Barrero, L.H., Lee, D.L., Dennerlein, J.T., 2007. Effect of horizontal position of the computer keyboard on upper extremity posture and muscular load during computer work. Ergonomics 50 (9), 1419-1432.

Lee, D.L., Kuo, P.L., Jindrich, D.L., Dennerlein, J.T. Computer keyswitch forcedisplacement characteristics affect muscle activity patterns during index finger tapping. Journal of Electromyography and Kinesiology, in press. doi:10.1016/j. jelekin.2008.03.011

Liang, H.W., Hwang, Y.H., Chang, F.H., 2008. Temporal change in bimanual interkeypress intervals and self-reported symptoms during continuous typing. Journal of Occupational Rehabilitation, doi:10.1007/s10926-008-9150-3.

Lin, M.I., Liang, H.W., Lin, K.H., Hwang, Y.H., 2004. Electromyographical assessment on muscular fatigue - an elaboration upon repetitive typing activity. Journal of Electromyography and Kinesiology 14, 661-669.

Mikkelsen, S., Vilstrup, I., Lassen, C.F., Kryger, A.I., Thomsen, J.F., Andersen, J.H., 2007. Validity of questionnaire self-reports on computer, mouse and keyboard usage during a four-week period. Occupational and Environmental Medicine 64, 541-547.

Murata, K., Araki, S., Kawakami, N., Saito, Y., Hino, E., 1991. Central nervous system effects and visual fatigue in VDT workers. International Archives of Occupational and Environmental Health 63 (2), 109-113.

Nakazawa, T. Okubo, Y, Suwazono, Y., Kobayashi, E, Komine, S., Kato, N., et al. 2002. Association between duration of daily VDT use and subjective symptoms. American Journal of Industrial Medicine 42 (5), 421-426.

Rempel, D., Serina, E., Klinenberg, E., Martin, B.J., Armstrong, T.J., Foulke, J.A., et al. 1997. The effect of keyboard keyswitch make force on applied force and finger flexor muscle activity. Ergonomics 40, 800-808.

Russinovich, M.E., Solomon, D.A., 2004. Microsoft ${ }^{\circledR}$ Windows ${ }^{\circledR}$ Internals, Microsoft Windows Server ${ }^{\mathrm{TM}}$ 2003, Windows XP, and Windows 2000, fourth ed. Microsoft Press, Redmond, WA.

Sauter, S.L., Schleifer, L.M., Knutson, S.J., 1991. Work posture, workstation design, and musculoskeletal discomfort in a VDT data entry task. Human Factors 33, 151-167.

Shaw, G.M., 2001. Adverse human reproductive outcomes and electromagnetic fields: a brief summary of the epidemiologic literature. Bioelectromagnetics, S5-S18.

Sillanpaa, J., Huikko, S., Nyberg, M., Kivi, P., Laippala, P., Uitti, J., 2003. Effect of work with visual display units on musculoskeletal disorders in the office environment. Occupational Medicine (Oxford) 53, 443-451.

Simoneau, G.G., Marklin, R.W., Berman, J.E., 2003. Effect of computer keyboard slope on wrist position and forearm electromyography of typists without musculoskeletal disorders. Physical Therapy 83 (9), 816-830.

Smith, M.J., 1997. Psychosocial aspects of working with video display terminals (VDTs) and employee physical and mental health. Ergonomics 40 (10) 1002-1015.

Wahlstrom, J., 2005. Ergonomics, musculoskeletal disorders and computer work. Occupational Medicine (Oxford) 55, 168-176. 